RUNNING HEAD: NEURAL BASIS FOR CONSUMER ETHNOCENTRISM

Re-submitted to: Neuroscience Letters (May 2020)

Word count (including abstract, main text, references, and figures legends): 4343

words

\title{
Grey matter volume and amplitude of low-frequency fluctuations predicts consumer ethnocentrism tendency
}

\author{
Jianping Huang ${ }^{1}$, Xiaoang Wan ${ }^{1}$, Kaiping Peng ${ }^{1}$, \& Jie Sui ${ }^{2}$
}

1. Department of Psychology, Tsinghua University, Beijing, China

2. Department of Psychology, University of Aberdeen, Aberdeen, UK

Correspondence:

Xiaoang Wan, Department of Psychology, Tsinghua University, Beijing, China 100084. Electronic mail: wanxa@mail.tsinghua.edu.cn Jie Sui, Department of Psychology, University of Aberdeen, Aberdeen, AB24 3FX, United Kingdom. Electronic mail: jie.sui@abdn.ac.uk 


\begin{abstract}
Consumer ethnocentrism tendency (CET) refers to consumers' belief about the appropriateness and morality of buying foreign products, and this concept characterizes consumers' tendency to differentiate in-group and out-group commercial products and to avoid imported products for nationalistic reasons. In order to identify the neural correlates of individual differences in CET, we conducted a combined voxel-based morphometry (VBM) and resting-state fMRI study with 228 healthy adults from mainland China, and examined the associations between selfreported CET scores and gray matter volume (GMV), as well as fractional amplitude of low-frequency fluctuations (fALFF). The VBM and fALFF results consistently associated consumer ethnocentrism with the middle temporal gyrus, and the fALFF results further revealed the roles of the anterior cingulate gyrus and anterior insula in CET. Collectively, these findings provide converging evidence about the neural correlates for dispositional attitudes toward domestic and foreign products.
\end{abstract}

Keywords: Ethnocentrism; attitude; VBM; fALFF; individual differences 


\section{Introduction}

With the advent of globalization and intense competition, it has become increasingly important to assess and understand consumers' attitude and preference toward domestic- and foreign-made commercial products. Consumers may hold a different belief about the appropriateness and morality of buying foreign products, and highly ethnocentric consumers consider the act of purchasing foreign-made products as an inappropriate and unpatriotic behavior [1]. Such attitudes are toward foreign-made products in general, which is different from boycotting purchasing products from a certain country [2].

Generally speaking, ethnocentrism refers to an individual's tendency to judge other people based on one's own group's values and standard, to view one's own group as superior, and to reject culturally dissimilar ideas [3]. Highly ethnocentric individuals take pride in the symbols and values of their ethnic or national groups while holding other groups' symbols and values in contempt [4]. The emergence and existence of ethnocentrism can be attributed to social identity processes, genetic similarity, kin recognition, and/or collectivism/individualism [5]. Numerous studies have demonstrated that people act more prosocially toward members of their own group than to those outside their group [6].

In order to assess consumers' attitudes toward commercial products, Shim and Sharma [1] derived a domain-specific concept from the general concept of ethnocentrism. The term of consumer ethnocentrism tendency (CET) was first used to refer to North American consumers' belief about the appropriateness and morality of 
buying foreign-made commercial products [1], and then generalized to a range of populations [7], including China [8]. Importantly, CET characterizes consumers' tendency to differentiate in-group and out-group commercial products and to avoid foreign products for nationalistic reasons [9]. For instance, highly ethnocentric consumers may consider purchasing imported products as being a wrongful behavior that can harm the domestic economy.

Recently, a meta-analysis of 60 studies with a total of more than fifteen thousand participants revealed a positive influence of CET on the evaluations of, and willingness to purchase, domestic products, as well as a negative influence of CET on the evaluation of foreign products [10]. Similar to many attitude constructs, CET is often accompanied with affinity for domestic products and aversion toward foreign products, cognitive bias in favor of domestic products' importance and superiority, and behavioral preference for domestic products over imported products [11].

Previous studies have examined the antecedents of CET [12]. Specifically, some studies have revealed the positive correlations between CET and collectivism [9, 13]. Collectivism (as contrasted to individualism) refers to cultural values about how people construe themselves and their relation to the surrounding world [14]. Collectivists consider people as interconnected to each other, whereas individualists consider people as independent of each other [15]. Therefore, people from collectivistic cultures tend to be more ingroup-oriented and perceive more differences between in-group and out-group members than those from individualistic cultures [16]. People who endorse collectivistic values would consider the influence of their 
actions on the group or society more, and show greater ethnocentric tendency than those who endorse individualistic values [9].

Numerous studies have documented different responses of human brains to ingroup and out-group people [17]. For example, a range of neural correlates are involved in the social categorization, action perception, empathy, and face perception of in-group members [18], including the medial prefrontal cortex, anterior insula, and anterior cingulate cortex [19, 20]. However, very few studies have examined the neural basis of different responses to domestic and foreign products. In a recent fMRI study, when the participants were looking at photos of domestic products and considering purchases, their CET level was positively correlated with the activation in certain brain areas such as the medial prefrontal cortex, middle temporal gyrus, and so on [21].

Considering that a combination of task-based fMRI, resting-state fMRI, and structural MRI studies can provide multi-modal evidence for the same variable of interest [22, 23], we conducted a combined voxel-based morphometry (VBM) and resting-state fMRI study to examine the structural and functional substrates for individual differences in CET. Using an automated user-independent voxel-wise approach, the VBM analyses allow us to associate individual differences in dispositional attitude or tendency to the gray matter volume (GMV) within certain brain areas $[24,25]$. Using indicators such as the fractional amplitude of lowfrequency fluctuation (fALFF) [26, 27], previous resting-state fMRI studies have also associated individual differences in empathy and pride to spontaneous neuronal 
activity at rest [28, 29]. Similarly, both the VBM and fALFF analyses can be used to investigate the individual differences in CET. Based on the previously documented prefrontal control of ethnocentrism [5] and the findings of the task-based fMRI study of CET [21], it seems reasonable to expect some associations between individual differences in CET and medial prefrontal cortex and/or middle temporal gyrus in the present study.

\section{Materials and methods}

\subsection{Participants}

A total of 228 healthy Chinese adults (mean age $=23.8 \pm 3.4$ years; ranging from 18 to 37 years; 115 women and 113 men) were recruited from the participants pool of the Psychology Department of Tsinghua University. This study was approved by the Human Research Ethics Committee of Center for Biomedical Imaging Research at Tsinghua University. All participants gave written informed consent before the study started, and received 100 Chinese Yuan for their participation.

\subsection{Assessment of consumer ethnocentrism}

Consumer ethnocentrism was measured using the Chinese version [30] of CET scale developed by Shimp and Sharma [1] for North American participants. In the Chinese version, nationality-related expressions such as Chinese and China replaced American and the USA, respectively, in the original version of the CET. The participants were asked to rate the extent to which they agree or disagree with each 
item on a 7-point Likert scale that ranges from "strongly disagree" to "strongly agree.” Each participant's responses of the 17 items were summed to obtain the total CET score which can range from 17 to 119, with larger scores indicating a greater ethnocentrism tendency. The CET scale is shown to be reliable in this study, Cronbach's $\alpha=0.922$.

\subsection{Structural MRI and resting-state fMRI data acquisition}

Both structural MRI and resting-state fMRI scanning were performed at the Center for Biomedical Imaging Research of Tsinghua University, using a Philips Achieva 3.0T TX system with a standard 8-channel head coil.

As for structural MRI data acquisition, high-resolution T1-weighted anatomical images were acquired with 160 contiguous sagittal slices (thickness $=1 \mathrm{~mm}$, and flip angle $=8^{\circ}$ ). The sensitivity encoding (SENSE) parallel-imaging technique was employed to reduce scan time [31]. The SENSE acceleration factor was set to be 2 for the anterior-posterior direction, and set to be 1.5 for the right-left direction. Repetition time was set to be $8.2 \mathrm{~ms}$, and echo time was set to be $3.8 \mathrm{~ms}$. The acquisition matrix was 256 voxels $\times 256$ voxels $\times 160$ voxels, and the voxel size was $0.938 \mathrm{~mm} \times 0.938$ $\mathrm{mm} \times 1 \mathrm{~mm}$.

During resting-state fMRI scanning, all participants were instructed to stay awake with their eyes closed, and to keep their bodies physically still without thinking about anything in particular. The scanning consisted of 221 contiguous EPI volumes with the following scanning parameters: $\mathrm{TE}=35 \mathrm{~ms}$; $\mathrm{TR}=2300 \mathrm{~ms}$; number of slices 
= 37; flip angle $=90^{\circ} ; \mathrm{FOV}=240 \times 240 \mathrm{~mm}^{2} ;$ thickness $=2.5 \mathrm{~mm}$; gap $=0.95 \mathrm{~mm}$; acquisition voxel size $=2.5 \times 2.5 \times 2.5 \mathrm{~mm}^{3}$. The total duration of the resting-state scan was $508 \mathrm{~s}$.

\subsection{Structural MRI data preprocessing and VBM analyses}

The preprocessing of the structural MRI data was conducted via the MATLAB R2013b software (Mathworks, USA) installed with SPM8 (Wellcome Trust Centre for Neuroimaging, UK). After each image had been displayed in SPM8 to screen for artifacts or gross anatomical abnormalities, T1-weighted anatomical registration was performed through Exponentiated Lie (DARTEL) algebra for registration, normalization, and modulation [32]. All T1 images were segmented into gray matter, white matter, and cerebrospinal fluid. Grey matter images were rigidly aligned and resampled to $1.5 \times 1.5 \times 1.5 \mathrm{~mm}^{3}$ and normalized to a template in MNI space. After that, we also smoothed the modulated gray matter images using an 8-mm full-width half-maximum Gaussian kernel to reduce spatial noise.

In order to investigate the neuroanatomical correlates of individual differences in consumer ethnocentrism tendency, we then performed a linear regression analysis on the participants' CET scores, with age, gender, and total intracranial volume as covariates. Based on Eklund et al.'s [33] suggestion, we assessed statistical significance at the cluster-level using the non-stationary random field theory [34]. Specifically, statistical maps were assessed at an uncorrected threshold of $p<0.001$, and clusters were considered as significant if they passed a cluster-level threshold of $p$ 
$<0.05$ after non-stationary cluster-level FWE correction.

\subsection{Resting-state fMRI data preprocessing and fALFF Analyses}

The preprocessing of the resting-state functional MRI data preprocessing was performed using the DPABI software [35] on the MATLAB R2018 platform. We discarded the first 10 volumes for signal equilibrium, whereas the rest of the volumes were corrected for differences in slice-acquisition timing and spatially realigned to correct for head motion. All participants' head motions were smaller than $2 \mathrm{~mm}$ in translation, $2^{\circ}$ in rotation, and $0.2 \mathrm{~mm}$ in frame-wise displacement (FD) throughout the resting-state scanning. We regressed out the head movement, white matted signals, cerebrospinal fluid signals, and linear drift as nuisance parameters. Then the images were spatially normalized to the standard Montreal Neurological Institute (MNI) space (voxel size: $3 \times 3 \times 3 \mathrm{~mm}^{3}$ ), and smoothed via a Gaussian kernel of 8-mm full width at half maximum (FWHM).

Similar to Fujino et al.'s [36] and Takeuchi et al.'s [37] studies, we conducted the calculation of fALFF using the DPABI software. The fALFF parameter represents the ratio of the power within the low-frequency range $(0.01-0.10 \mathrm{~Hz})$ to the power of the entire frequency band $(0-0.25 \mathrm{~Hz})$ [27]. The fALFF maps were registered to the MNI 152 space, and individual fALFF maps were normalized to Z-maps for standardization purposes.

In order to find the brain regions whose fALFF values were significantly correlated to individual differences in CET, we performed a whole-brain analysis on 
the fALFF values using a general linear model (GLM) with the participants' CET scores being the covariates of interest while age and gender being the confounding covariates. Statistical maps were assessed at an uncorrected threshold of $p<0.001$, and clusters were considered as being significant if they passed a cluster-level threshold of $\mathrm{p}<0.05$ after Gaussian random field (GRF) correction.

\section{Results}

First, descriptive analyses showed that the participants’ CET scores are normally distributed (skewness $=0.38$, kurtosis $=-0.48)$, with a scoring range of 17 to $77,(\mathrm{M}=$ 39.67, $\mathrm{SD}=14.43)$. An independent-samples $t$ test revealed that the male participants $(\mathrm{M}=42.11, \mathrm{SD}=15.00)$ scored significantly higher than the female participants $(\mathrm{M}=$ 37.27, $\mathrm{SD}=13.49), t(226)=2.56, p=0.011$, Cohen's $d=0.34$.

Second, the whole-brain VBM analysis revealed significant positive correlations between the participants' CET scores and the GMV in the right middle temporal gyrus (peak MNI coordinates [59, 6, -29], $t=4.09, k=156, p=0.03$ ), as well as the GMV in the left middle frontal gyrus (peak MNI coordinates [-36, 12, 41], $t=4.18, k=143$, $p=0.051$, after non-stationary cluster-level FWE correction. As shown in Figure 1, the partial correlation analyses revealed that the participants' CET scores were positively with the GMV in the right middle temporal gyrus, $r=0.27, p<0.001$, and the GMV in the left middle frontal gyrus, $r=0.30, p<0.001$, 


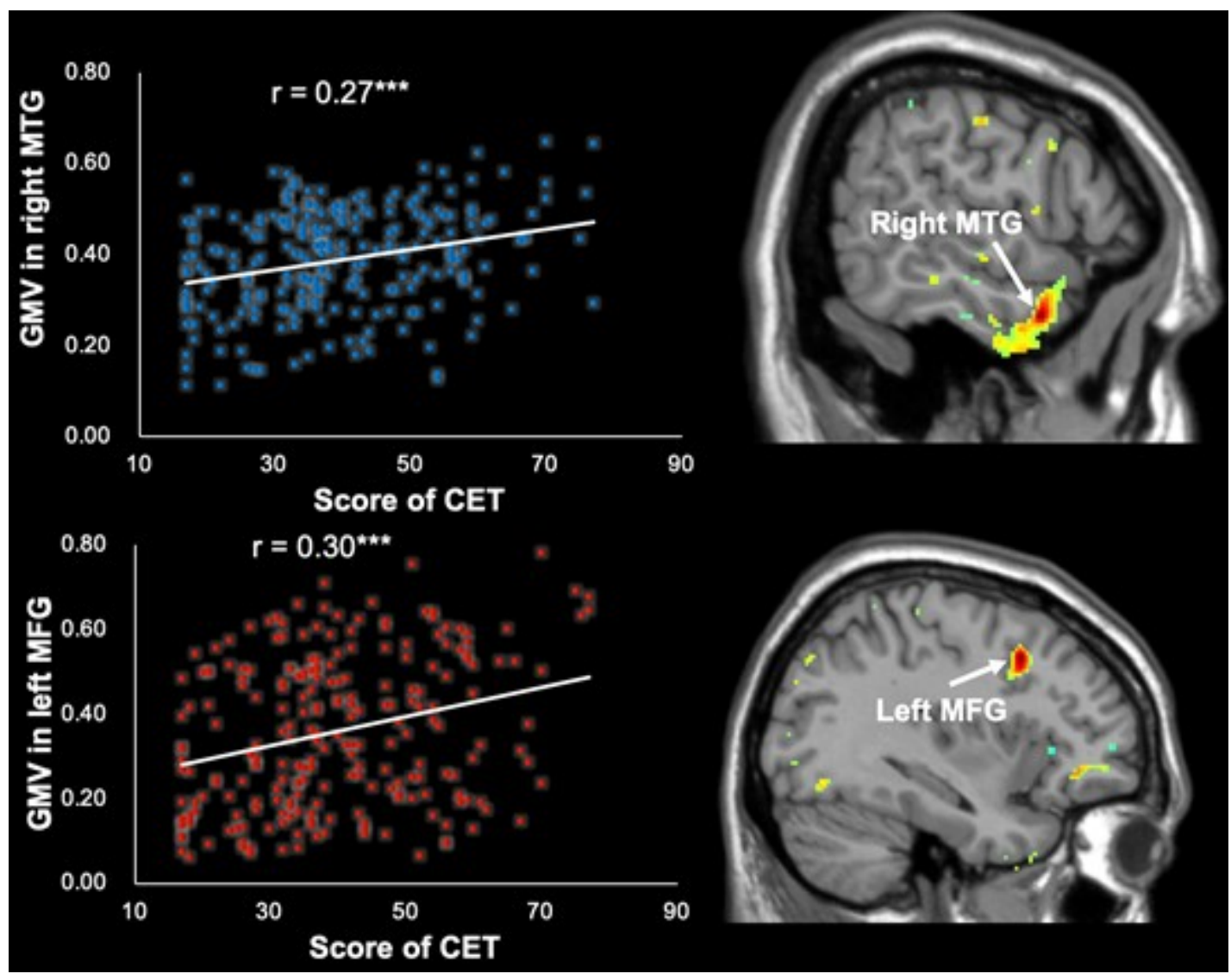

Figure 1. The participants' CET scores were positively correlated with the GMV in the right middle temporal gyrus (MTG; upper panel) and that in the left middle frontal gyrus (MFG; lower panel). Note that *** denotes $p<.001$.

Third, the whole-brain analysis on the fALFF parameters revealed significant positive correlations between the participants' CET scores and the fALFF in the left middle temporal gyrus (peak MNI coordinates [-57, -12, -33], $t=4.44, k=25$ ), the left anterior cingulate gyrus (peak MNI coordinates [-6, 27, -15], $t=3.54, k=10$ ), and the left anterior insula (peak MNI coordinates [-33, 9, 18], $t=3.93, k=36$ ), after GRF correction. As shown in Figure 2, the participants' CET scores were positively correlated with the fALFF in the left middle temporal gyrus, $r=0.28, p<0.001$, the 
fALFF in the left anterior cingulate gyrus, $r=0.19, p=0.004$, and the fALFF in the left anterior insula, $r=0.22, p=0.001$.

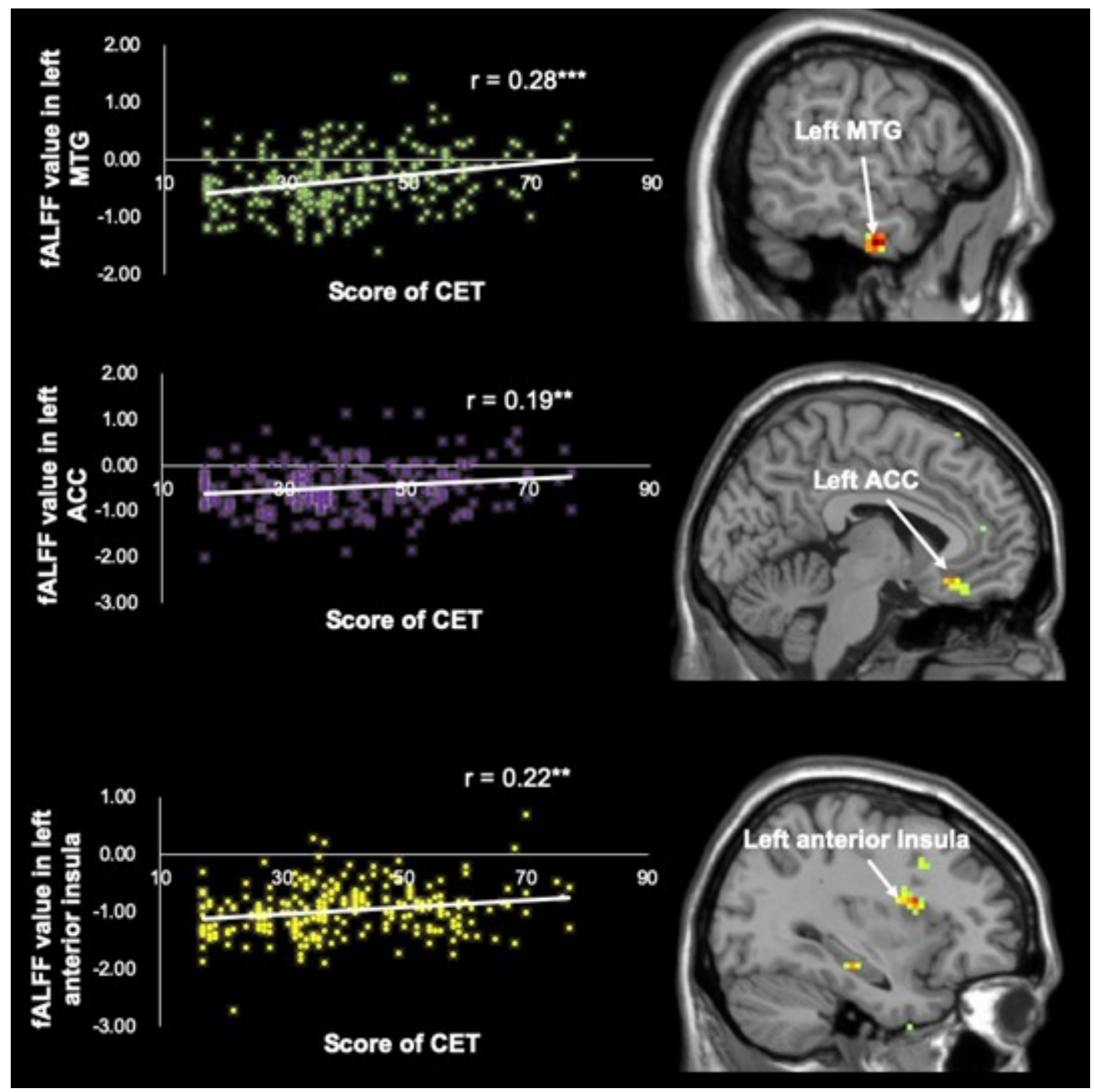

Figure 2. The fALFF values of three regions were positively correlated with the participants' CET scores, including the left middle temporal gyrus (MTG; upper panel), the left anterior cingulate gyrus (ACC; middle panel), and the left anterior insula (lower panel). Note that $* *$ denotes $p<.01$, and $* * *$ denotes $p<.001$.

\section{Discussion}


In summary, three major results emerged from the present study. First, the results of our VBM analyses revealed positive correlations between the participants' CET scores and the GMV in the right middle temporal gyrus and the left middle frontal gyrus. Previous research has demonstrated the prefrontal control of ethnocentrism in general [5], and a recent fMRI study has associated consumer ethnocentrism to activation in the medial prefrontal cortex and middle temporal gyrus [21]. Even though our results do not reveal any significant correlations between individual differences in CET and medial prefrontal cortex, our findings do provide converging multi-modal evidence regarding the association between the middle temporal gyrus and consumer ethnocentrism. Considering the positive associations between collectivism and consumer ethnocentrism [9, 13], our findings are in line with the literature that associated collectivism to the middle temporal gyrus [38] and the middle frontal gyrus [39]. A greater volume of gray matter within a certain brain area often implies better computational efficacy and stronger processing ability [40]. Therefore, our findings suggest that the middle temporal gyrus and the middle frontal gyrus may be involved in the neural substrates of consumer ethnocentrism due to their associations with collectivism.

Second, the results of our fALFF analyses associated consumer ethnocentrism to the left middle temporal gyrus, the left anterior cingulate gyrus, and the left anterior insula. The fALFF results of the left middle temporal gyrus are in line with the VBM results of the right middle temporal gyrus, and consistently link the middle temporal gyrus to consumer ethnocentrism [21]. Moreover, the fALFF results of the anterior 
cingulate gyrus are in line with Volz et al.'s [19] findings that greater activation within the anterior cingulate cortex was observed when participant showed in-group favoritism. As an index of spontaneous signal fluctuations, a higher value of fALFF indicates stronger associations with the BOLD signal [41] and regional metabolic rate [42]. Thus, the positive correlations between the fALFF value of the left anterior cingulate gyrus and the participants' CET scores suggest that a higher level of ethnocentrism may be associated with the more excitatory intrinsic activity in this brain region. Furthermore, previous research on intergroup empathy has also identified the role of anterior insula when the participants showed preferential neural responses when perceiving the pain of in-group members compared to out-group members [20]. Therefore, the anterior insula may be involved in the neural substrates of consumer ethnocentrism due to its role in emotion-related processing related to ingroup bias.

Third, our psychometric data suggest that Chinese consumers' ethnocentrism tendency varies from one individual to another (ranging from 17 to 77 points on a 17item 7-point scale). In addition to individual differences, our results also revealed a significant gender difference with male participants scoring higher than females. It should be noted that there has been mixed evidence about the gender difference in CET [13]. For example, one study has shown that women were more ethnocentric than men based on the data collected in Korea [9]; whereas another study has suggested that men were more ethnocentric than women based on the data collected in the UK [43]. Collectively, these results suggest that gender difference in consumer 
ethnocentrism may not be universal across cultures, and such cross-cultural differences in the gender differences in CET cannot be simply attributed to the differentiation between Eastern and Western, or between collectivist and individualistic cultures.

As with any study, there are also certain limitations in the present study as far as interpretation and generalization are concerned. First, we only tested participants from mainland China, whereas previous research has documented come cross-cultural differences in consumer ethnocentrism [44, 45]. Therefore, cautions are called for if one tries to generalize our results with Chinese participants directly to other populations. Second, we only used self-reported scale to measure the participants' ethnocentrism tendency in the present study, but it will be very interesting to associate participants' ratings of, and willingness to purchase domestic and foreign products with brain structural differences and/or spontaneous neuronal activity in future research.

In conclusion, the findings of the present study associate individual differences in consumer ethnocentrism tendency to several brain areas that have been linked to collectivism and in-group favoritism in previous research. The results of our VBM analyses associated individual differences in consumers' attitude toward domestic and foreign products with structural variations within the middle frontal gyrus and the middle temporal gyrus. The results of our fALFF analyses associated different attitudes toward domestic and foreign products to the spontaneous brain activity in the middle temporal gyrus, anterior cingulate gyrus, and anterior insula at rest. 
Collectively, these findings provide converging evidence about the neural correlates for dispositional attitudes. Considering that consumer ethnocentrism can result in the sense of identity and a feeling of belongings [1], our findings may also provide some insight into the neural substrate of social perception in general. 


\section{References}

1. Shimp, T. A., \& Sharma, S. (1987). Consumer ethnocentrism: Construction and validation of the CETSCALE. Journal of Marketing Research, 24(8), 280-289.

2. Meng, J., Meng, Y., \& Liu, M. T. (2012). The impact of consumer animosity on country-of-origin effect: Evidence from the political event of Chinese opposing Japan’s UN bid. Journal of Euromarketing, 21(4), 219-227.

3. Booth, K. (1979). Strategy and ethnocentrism. London: Croom-Helm.

4. Levin, R. A., \& Campbell, D. T. (1972). Ethnocentrism: Theories of conflict, ethnic attitudes, and group behavior. New Yoke: John Wiley \& Sons.

5. MacDonald, K. B. (2008). Effortful control, explicit processing, and the regulation of human evolved predispositions. Psychological Review, 115(4), 1012-1031.

6. Everett, J. A., C., Faber, N. S., \& Crockett, M. (2015). Preferences and beliefs in ingroup favoritism. Frontiers in Behavioral Neuroscience, 9: 5.

7. Netemeyer, R. G., Durvasula, S., \& Lichtenstein, D. R. (1991). A cross-national assessment of the reliability and validity of the CETSCALE. Journal of Marketing Research, 28(3), 320-327.

8. Wang, C. L., \& Chen, Z. X. (2004). Consumer ethnocentrism and willingness to buy domestic products in a developing country setting: Testing moderating effects. Journal of Consumer Marketing, 21, 391-400.

9. Sharma, S., Shimp, T. A., \& Shin, J. (1995). Consumer ethnocentrism: A test of antecedents and moderators. Journal of the Academy of Marketing Science, 23(1), 26-37.

10. Guo, G., \& Zhou, X. (2017). Consumer Ethnocentrism on product judgment and willingness to buy: A meta-analysis. Social Behavior and Personality, 45(1), 163-176.

11. Sharma, S. (2015). Consumer ethnocentrism: Reconceptualization and crosscultural validation. Journal of International Business Studies, 46(3), 381-389.

12. Shankarmahesh, M. N. (2006). Consumer ethnocentrism: An integrative review 
of its antecedents and consequences. International Marketing Review, 23(2), $146-172$.

13. Nishina, S. (1990). Japanese consumers: Introducing foreign products/brands into the Japanese market. Journal of Advertising Research, 30(2), 35-45.

14. Triandis, H. C. (1995). Individualism and collectivism. Boulder, CO: Westview Press.

15. Markus, H., \& Kitayama, S. (1991). Culture and the self: Implication for cognition, emotion, and motivation. Psychological Review, 98, 224-253.

16. Triandis, H. C., \& Trafimow, D. (2003). Culture and its implications for intergroup behavior. In R. Brown \& S. L. Gaertner (Eds.), Blackwell handbook of social psychology: Intergroup processes (pp. 367-385). Oxford: Blackwell Publishers Ltd.

17. Ito, T. A., \& Bartholow, B. D. (2009). The neural correlates of race. Trends in Cognitive Sciences, 13, 524-531.

18. Molenberghs, P. (2013). The neuroscience of in-group bias. Neuroscience and Biobehavioral Reviews, 37, 1530-1536.

19. Volz, K. G., Kessler, T., \& Von Cramon, D. Y. (2009). In-group as part of the self: In-group favoritism is mediated by medial prefrontal cortex activation. Social Neuroscience, 4, 244-260.

20. Xu, X., Zuo, X., Wang, X., \& Han, S. (2009). Do you feel my pain? Racial group membership modulates empathic neural responses. Journal of Neuroscience, 29, 8525-8529.

21. Casado-Aranda, L. A., Sánchez-Fernández, J., Ibáñez-Zapata, J. Á., \& LiébanaCabanillas, F. J. (2020). How consumer ethnocentrism modulates neural processing of domestic and foreign products: A neuroimaging study. Journal of Retailing and Consumer Services, 53: 101961.

22. Fox, M. D., \& Raichle, M. E. (2007). Spontaneous fluctuations in brain activity observed with functional magnetic resonance imaging. Nature Reviews Neuroscience, 8(9), 700-711.

23. Logothetis, N. K., Pauls, J., Augath, M., Trinath, T., \& Oeltermann, A. (2001). 
Neurophysiological investigation of the basis of the fMRI signal. Nature, 412, $150-157$.

24. Liu, H., Wang, Y., Liu, W., Wei, D., \& Yang, J. (2016). Neuroanatomical correlates of attitudes toward suicide in a large healthy sample: A voxel-based morphometric analysis. Neuropsychologia, 80, 185-193.

25. Haas, B. W., Ishak, A., Anderson, I. W., \& Filkowski, M. M. (2015). The tendency to trust is reflected in human brain structure. NeuroImage, 107, 175181.

26. Zang, Y., He, Y., Zhu, C., Cao, Q., Sui, M., \& Liang, M. et al. (2007). Altered baseline brain activity in children with ADHD revealed by resting-state functional MRI. Brain and Development, 29(2), 83-91.

27. Zou, Q., Zhu, C., Yang, Y., Zuo, X., Long, X., \& Cao, Q. et al. (2008). An improved approach to detection of amplitude of low-frequency fluctuation (ALFF) for resting-state fMRI: Fractional ALFF. Journal of Neuroscience Methods, 172, 137-141.

28. Cox, C. L., Uddin, L. Q., Di Martino, A., Castellanos, F. X., \& Milham, M. P., \& Kelly, C. (2012). The balance between feeling and knowing: Affective and cognitive empathy are reflected in the brain's intrinsic functional dynamics. Social Cognitive and Affective Neuroscience, 7, 727-737.

29. Kong, F., He, Q., Liu, X., Chen, X., Wang, X., \& Zhao, J. (2018). Amplitude of low-frequency fluctuations during resting state differentially predicts authentic and hubristic pride. Journal of Personality, 86(2), 213-219.

30. Wang, H. (2003). Consumer ethnocentrism: An empirical test in China and its marketing managerial implications. Nankai Business Review, 2003(4), 31-26. [in Chinese].

31. Pruessmann, K. P., Weiger, M., Scheidegger, M. B., \& Boesiger, P. (1999). SENSE: Sensitivity encoding for fast MRI. Magnetic Resonance in Medicine, 42(5), 952-962.

32. Ashburner, J. (2007). A fast diffeomorphic image registration algorithm. NeuroImage, 38(1), 95-113. 
33. Eklund, A., Nichols, T. E., \& Knutsson, H. (2016). Cluster failure: Why fMRI inferences for spatial extent have inflated false-positive rates. Proceedings of the National Academy of Sciences, 113(28), 7900-7905

34. Hayasaka, S., Phan, K. L., Liberzon, I., Worsley, K. J., \& Nichols, T. E. (2004). Nonstationary cluster-size inference with random field and permutation methods. NeuroImage, 22(2), 676-687.

35. Yan, C., Wang, X. Zuo, X., \& Zang, Y. (2016). DPABI: Data processing and analysis for (resting-state) brain imaging. Neuroinformatics, 14, 339-351.

36. Fujino, J., Tei, S., Jankowski, K. F., Kawada, R., Murai, T., \& Takahashi, H. (2017). Role of spontaneous brain activity in explicit and implicit aspects of cognitive flexibility under socially conflicting situations: A resting-state fMRI study using fractional amplitude of low-frequency fluctuations. Neuroscience, 367, 60-71.

37. Takeuchi, H., Taki, Y., Nouchi, R., Yokoyama, R., Kotozaki, Y., \& Nakagawa, S., et al. (2017). Regional homogeneity, resting-state functional connectivity and amplitude of low frequency fluctuation associated with creativity measured by divergent thinking in a sex-specific manner. NeuroImage, 152, 258-269

38. Chiao, J. Y., Harada, T., Komeda, H., Li, Z., Mano, Y., \& Saito, D. et al. (2009). Neural basis of individualistic and collectivistic views of self. Human Brain Mapping, 30, 2813-2820.

39. Lin, Y. C., Qu, Y., \& Telzer, E. H. (2018). Cultural influences on the neural correlates of intergroup perception. Culture and Brain, 6,171-187.

40. Kanai, R., \& Rees, G. (2011). The structural basis of inter-individual differences in human behaviour and cognition. Nature Reviews Neuroscience, 12(4), 231242.

41. Li, Y. C., Chen, C. C., \& Chen, J. H. (2011). Impact of visual repetition rate on intrinsic properties of low frequency fluctuations in the visual network. PloS One, 6(5): e18954.

42. Tomasi, D., Wang, G. J., \& Volkow, N. D. (2013). Energetic cost of brain functional connectivity. Proceedings of the National Academy of Sciences, 
110(33), 13642-13647.

43. Bannister, J. P., \& Saunders, J. A. (1978). UK consumers' attitudes towards imports: The measurement of national stereotype image. European Journal of Marketing, 12, 562-570.

44. Tsai, W. S., Lee, W.-N., \& Yoo, J. J. (2013). A cross-cultural study of consumer ethnocentrism between China and the U.S. Journal of International Consumer Marketing, 25, 80-93.

45. Tsai, W. S., Yoo, J. J., \& Lee, W.-N. (2013). For love of country? Consumer ethnocentrism in China, South Korea, and the United States. Journal of Global Marketing, 26, 98-114. 


\section{Acknowledgements}

This work was supported by the National Natural Science Foundation of China (Grant No. 71872097) awarded to Xiaoang Wan. Comments concerning this article should be sent to Prof. Xiaoang Wan at wanxa@mail.tsinghua.edu.cn or Prof. Jie Sui at jie.sui@abdn.ac.uk.

\section{Conflict of Interest Statement}

None. 